\title{
A very-high-energy component deep in the Gamma-ray Burst afterglow
}

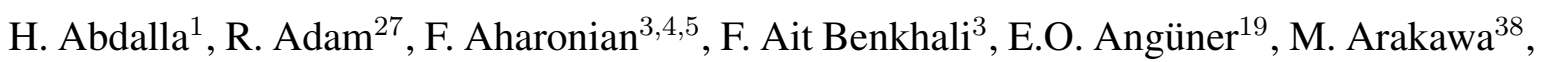

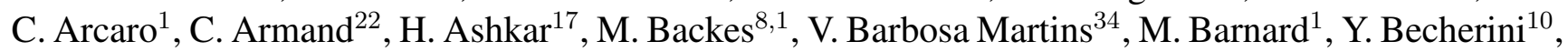
D. Berge ${ }^{\sqrt[34]{4}}$, K. Bernlöht ${ }^{3}$, E. Bissaldi ${ }^{4544}$, R. Blackwell ${ }^{13}$, M. Böttcher ${ }^{11}$, C. Boisson ${ }^{14}$, J. Bolmont ${ }^{15}$,

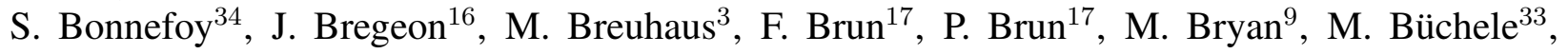

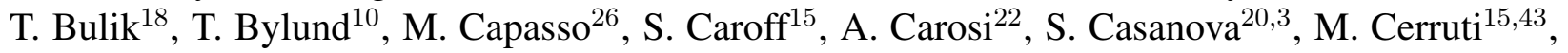
T. Chand ${ }^{1}$, S. Chandra ${ }^{1}$, A. Chen ${ }^{21}$, S. Colafrancesco ${ }^{21} 2$, M. Curyło ${ }^{18}$, I.D. Davids ${ }^{\sqrt[8]{6}}$, C. Deil ${ }^{3}$,

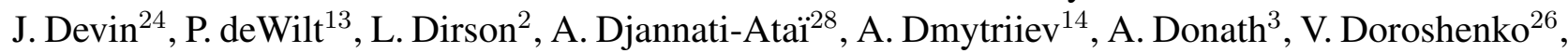

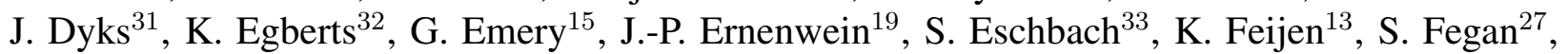
A. Fiasson ${ }^{22}$, G. Fontaine ${ }^{27}$, S. Funk ${ }^{33}$, M. Füßling 3 , S. Gabici28, Y.A. Gallant ${ }^{16}$, F. Gate $e^{22}$, G. Giavitto ${ }^{34}$, L. Giunti ${ }^{28}$, D. Glawion ${ }^{23}$, J.F. Glicenstein 17 , D. Gottschall ${ }^{26}$, M.-H. Grondin 24 , J. Hahn ${ }^{3}$, M. Haup ${ }^{\sqrt[34]{4}}$, G. Heinzelmann ${ }^{2}$, G. Henri ${ }^{29}$, G. Hermann ${ }^{3}$, J.A. Hinton ${ }^{3}$, W. Hofmann ${ }^{3}$,

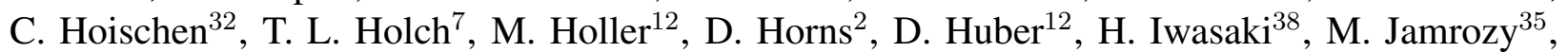
D. Jankowsky $\sqrt{33}$, F. Jankowsky ${ }^{23}$, A. Jardin-Blicq ${ }^{3}$, I. Jung-Richardt ${ }^{33}$, M.A. Kastendieck ${ }^{2}$, K. Katarzyński ${ }^{36}$,

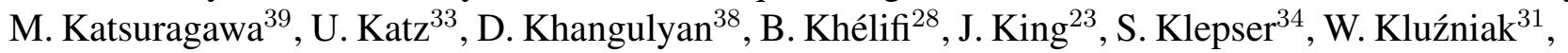
Nu. Komin ${ }^{21}$, K. Kosack 17 , D. Kostunin ${ }^{34}$, M. Kreter ${ }^{11}$, G. Lamanna ${ }^{22}$, A. Lemière ${ }^{28}$, M. Lemoine-

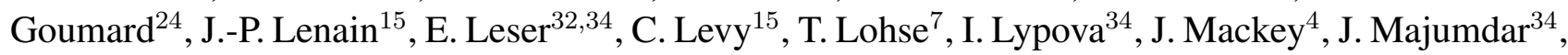
D. Malyshev $\sqrt{26}$, V. Marandon ${ }^{3}$, A. Marcowith ${ }^{16}$, A. Mares ${ }^{24}$, C. Mariaud 27, G. Martí-Devesa ${ }^{12}$, R. Marx ${ }^{[3}$, G. Maurin ${ }^{22}$, P.J. Meintjes $\frac{37}{37}$ A.M.W. Mitchell[342, R. Moderski 31 , M. Mohamed ${ }^{23}$,

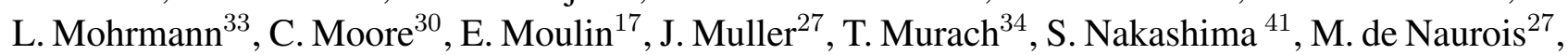

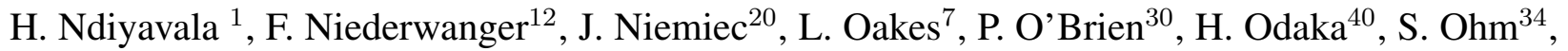
E. de Ona Wilhelmit ${ }^{34}$, M. Ostrowski ${ }^{\sqrt{35}}$, I. Oya ${ }^{34}$, M. Panter ${ }^{3}$, R.D. Parsons ${ }^{3}$, C. Perennes ${ }^{15}$, P.-

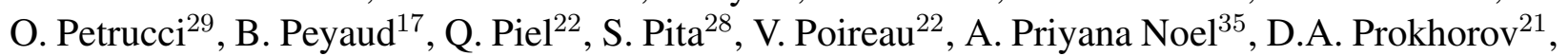
H. Prokoph ${ }^{34}$, G. Pühlhofer ${ }^{26}$, M. Punch $\frac{28 \mid 10}{}$, A. Quirrenbach ${ }^{23}$, S. Raab ${ }^{33}$, R. Rauth ${ }^{12}$, A. Reimer ${ }^{12}$, O. Reimer ${ }^{12}$, Q. Remy ${ }^{16}$, M. Renaud ${ }^{16}$, F. Rieger ${ }^{3}$, L. Rinchiusd ${ }^{17}$, C. Romoli ${ }^{3}$, G. Rowell ${ }^{13}$, B. Rudak ${ }^{31}$, E. Ruiz-Velasco ${ }^{3}$, V. Sahakian ${ }^{6}$, S. Sailer $^{3}$, S. Saito ${ }^{38}$, D.A. Sanchez ${ }^{22}$, A. Santangeld ${ }^{26}$,

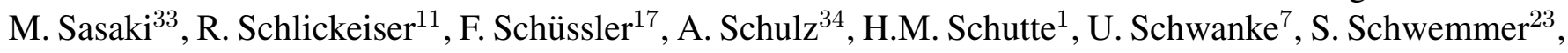
M. Seglar-Arroyd ${ }^{17}$, M. Senniappan ${ }^{10}$, A.S. Seyffer ${ }^{11}$, N. Shafi $\stackrel{21}{ }$, K. Shiningayamwe ${ }^{8}$, R. Simoni ${ }^{9}$,

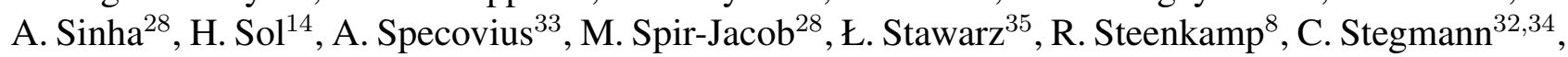

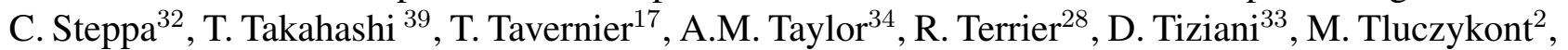
C. Trichard ${ }^{27}$, M. Tsirou ${ }^{16}$, N. Tsuji ${ }^{38}$, R. Tuffs ${ }^{3}$, Y. Uchiyama $^{38}$, D.J. van der Wal ${ }^{11}$, C. van Eldik ${ }^{33}$,

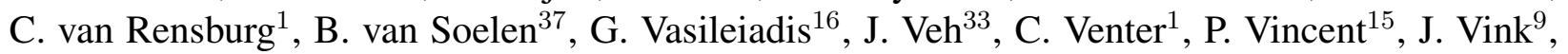
H.J. Völk ${ }^{3}$, T. Vuillaume ${ }^{22}$, Z. Wadiasingh ${ }^{1}$, S.J. Wagner ${ }^{23}$, R. White ${ }^{3}$, A. Wierzcholska ${ }^{20 \mid 23}$,

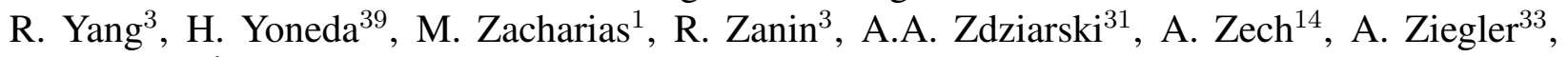
J. Zorn ${ }^{3}$, N. Żywucka ${ }^{1}$, F. de Palma ${ }^{46}$, M. Axelsson $25 \mid 47$, O.J. Roberts $\stackrel{48}{4}$

${ }^{1}$ Centre for Space Research, North-West University, Potchefstroom 2520, South Africa

${ }^{2}$ Universität Hamburg, Institut für Experimentalphysik, Luruper Chaussee 149, D 22761 Hamburg, Germany

${ }^{3}$ Max-Planck-Institut für Kernphysik, P.O. Box 103980, D 69029 Heidelberg, Germany 
${ }^{4}$ Dublin Institute for Advanced Studies, 31 Fitzwilliam Place, Dublin 2, Ireland

${ }^{5}$ High Energy Astrophysics Laboratory, RAU, 123 Hovsep Emin St Yerevan 0051, Armenia

${ }^{6}$ Yerevan Physics Institute, 2 Alikhanian Brothers St., 375036 Yerevan, Armenia

${ }^{7}$ Institut für Physik, Humboldt-Universität zu Berlin, Newtonstr. 15, D 12489 Berlin, Germany

${ }^{8}$ University of Namibia, Department of Physics, Private Bag 13301, Windhoek, Namibia, 12010

${ }^{9}$ GRAPPA, Anton Pannekoek Institute for Astronomy, University of Amsterdam, Science Park 904, 1098 XH Amsterdam, The Netherlands

${ }^{10}$ Department of Physics and Electrical Engineering, Linnaeus University, 35195 Växjö, Sweden

${ }^{11}$ Institut für Theoretische Physik, Lehrstuhl IV: Weltraum und Astrophysik, Ruhr-Universität Bochum, D 44780 Bochum, Germany

${ }^{12}$ Institut für Astro- und Teilchenphysik, Leopold-Franzens-Universität Innsbruck, A-6020 Innsbruck, Austria

${ }^{13}$ School of Physical Sciences, University of Adelaide, Adelaide 5005, Australia

${ }^{14}$ LUTH, Observatoire de Paris, PSL Research University, CNRS, Université Paris Diderot, 5 Place Jules Janssen, 92190 Meudon, France

${ }^{15}$ Sorbonne Université, Université Paris Diderot, Sorbonne Paris Cité, CNRS/IN2P3, Laboratoire de Physique Nucléaire et de Hautes Energies, LPNHE, 4 Place Jussieu, F-75252 Paris, France

${ }^{16}$ Laboratoire Univers et Particules de Montpellier, Université Montpellier, CNRS/IN2P3, CC 72, Place Eugène Bataillon, F-34095 Montpellier Cedex 5, France

${ }^{17}$ IRFU, CEA, Université Paris-Saclay, F-91191 Gif-sur-Yvette, France

${ }^{18}$ Astronomical Observatory, The University of Warsaw, Al. Ujazdowskie 4, 00-478 Warsaw, Poland

${ }^{19}$ Aix Marseille Université, CNRS/IN2P3, CPPM, Marseille, France

${ }^{20}$ Instytut Fizyki Jạdrowej PAN, ul. Radzikowskiego 152, 31-342 Kraków, Poland

${ }^{21}$ School of Physics, University of the Witwatersrand, 1 Jan Smuts Avenue, Braamfontein, Johannesburg, 2050 South Africa

${ }^{22}$ Laboratoire d'Annecy de Physique des Particules, Univ. Grenoble Alpes, Univ. Savoie Mont Blanc, CNRS, LAPP, 74000 Annecy, France

${ }^{23}$ Landessternwarte, Universität Heidelberg, Königstuhl, D 69117 Heidelberg, Germany

${ }^{24}$ Université Bordeaux, CNRS/IN2P3, Centre d'Études Nucléaires de Bordeaux Gradignan, 33175 Gradignan, France

${ }^{25}$ Oskar Klein Centre, Department of Physics, Stockholm University, Albanova University Center, SE10691 Stockholm, Sweden

${ }^{26}$ Institut für Astronomie und Astrophysik, Universität Tübingen, Sand 1, D 72076 Tübingen, Germany

${ }^{27}$ Laboratoire Leprince-Ringuet, cole Polytechnique, UMR 7638, CNRS/IN2P3, Institut Polytechnique de Paris, F-91128 Palaiseau, France

${ }^{28}$ APC, AstroParticule et Cosmologie, Université Paris Diderot, CNRS/IN2P3, CEA/Irfu, Observatoire de Paris, Sorbonne Paris Cité, 10, rue Alice Domon et Léonie Duquet, 75205 Paris Cedex 13, France

${ }^{29}$ Univ. Grenoble Alpes, CNRS, IPAG, F-38000 Grenoble, France

${ }^{30}$ Department of Physics and Astronomy, The University of Leicester, University Road, Leicester, LE1 7RH, United Kingdom

${ }^{31}$ Nicolaus Copernicus Astronomical Center, Polish Academy of Sciences, ul. Bartycka 18, 00-716 Warsaw, Poland

${ }^{32}$ Institut für Physik und Astronomie, Universität Potsdam, Karl-Liebknecht-Strasse 24/25, D 14476 Potsdam, Germany

${ }^{33}$ Friedrich-Alexander-Universität Erlangen-Nürnberg, Erlangen Centre for Astroparticle Physics, Erwin- 
Rommel-Str. 1, D 91058 Erlangen, Germany

${ }^{34}$ DESY, D-15738 Zeuthen, Germany

${ }^{35}$ Obserwatorium Astronomiczne, Uniwersytet Jagielloński, ul. Orla 171, 30-244 Kraków, Poland

${ }^{36}$ Centre for Astronomy, Faculty of Physics, Astronomy and Informatics, Nicolaus Copernicus University, Grudziadzka 5, 87-100 Torun, Poland

${ }^{37}$ Department of Physics, University of the Free State, PO Box 339, Bloemfontein 9300, South Africa

${ }^{38}$ Department of Physics, Rikkyo University, 3-34-1 Nishi-Ikebukuro, Toshima-ku, Tokyo 171-8501, Japan

${ }^{39}$ Kavli Institute for the Physics and Mathematics of the Universe (WPI), The University of Tokyo Institutes for Advanced Study (UTIAS), The University of Tokyo, 5-1-5 Kashiwa-no-Ha, Kashiwa City, Chiba, 2778583, Japan

${ }^{40}$ Department of Physics, The University of Tokyo, 7-3-1 Hongo, Bunkyo-ku, Tokyo 113-0033, Japan

${ }^{41}$ RIKEN, 2-1 Hirosawa, Wako, Saitama 351-0198, Japan

${ }^{42}$ Physik Institut, Universität Zürich, Winterthurerstrasse 190, CH-8057 Zürich, Switzerland

${ }^{43}$ Institut de Ciències del Cosmos (ICC UB), Universitat de Barcelona (IEEC-UB), Martí Franquès 1, E08028 Barcelona, Spain

${ }^{44}$ Dipartimento Interateneo di Fisica, Politecnico di Bari, Via E. Orabona 4, 70125 Bari, Italy

${ }^{45}$ Istituto Nazionale di Fisica Nucleare - Sezione di Bari, Via E. Orabona 4, 70125 Bari, Italy

${ }^{46}$ Istituto Nazionale di Fisica Nucleare - Sezione di Torino, Via P. Giuria 1, 10125 Torino, Italy

${ }^{47}$ Department of Physics, KTH Royal Institute of Technology, AlbaNova, SE-106 91 Stockholm, Sweden

${ }^{48}$ Science and Technology Institute, Universities Space Research Association, 320 Sparkman Drive, Huntsville, AL 35805, USA

Gamma-ray bursts (GRBs) are brief flashes of gamma rays, considered to be the most energetic explosive phenomena in the Universe ${ }^{1}$. The emission from GRBs comprises a short (typically tens of seconds) and bright prompt emission, followed by a much longer afterglow phase. During the afterglow phase, the shocked outflow-produced by the interaction between the ejected matter and the circumburst medium-slows down,and a gradual decrease in brightness is observed ${ }^{2}$. GRBs typically emit most of their energy via $\gamma$-rays with energies in the kiloelectronvolt-to-megaelectronvolt range, but a few photons with energies of tens of gigaelectronvolts have been detected by space-based instruments ${ }^{3}$. However, the origins of such high-energy (above one gigaelectronvolt) photons and the presence of very-high-energy (more than 100 gigaelectronvolts) emission have remained elussive ${ }^{4}$. Here we report observations of very-high-energy emission in the bright GRB 180720B deep in the GRB afterglowten hours after the end of the prompt emission phase, when the X-ray flux had already decayed by four orders of magnitude. Two possible explanations exist for the observed radiation: inverse Compton emission and synchrotron emission of ultrarelativistic electrons. Our observations show that the energy fluxes in the $X$-ray and -ray range and their photon indices remain comparable to each other throughout the afterglow. This discovery places distinct constraints on the GRB environment for both emission mechanisms, with the inverse Compton explanation alleviating the particle energy requirements for the emission observed at late times. The late timing of this detection has consequences for the future observations of GRBs at the highest energies.

On July 20th 2018, GRB 180720B triggered the Fermi Gamma-ray Burst Monitor (GBM) at 14:21:39.65 UT ${ }^{5}\left(T_{0}\right)$ and the Swift Burst Alert Telescope (BAT) 5 s later ${ }^{6}$. Multi-wavelength 
follow-up observations were performed up to $T_{0}+3 \times 10^{5} \mathrm{~s}$, notably by the ESO Very Large Telescope (VLT) which measured a redshift $z=0.653^{\text {I }}$. In the high-energy (HE) gamma-ray band $(100 \mathrm{MeV}-100 \mathrm{GeV})$ this GRB was also detected by the Fermi Large Area Telescope (LAT) between $T_{0}$ and $T_{0}+700 \mathrm{~s}$ with a maximum photon energy of $5 \mathrm{GeV}$ at $T_{0}+142.4 \mathrm{~s}{ }^{\text {8 }}$. No further $\mathrm{HE}$ emission was detected in the successive observation windows after $700 \mathrm{~s}$. The prompt emission phase of GRB 180720B is extremely bright, ranking seventh in brightness among the over $\sim 2650$ GRBs detected by the Fermi-GBM up to date (see Methods). With a $T_{90}$ (the time in which $90 \%$ of the flux is detected) of $48.9 \pm 0.4 \mathrm{~s}$, it is categorised as a long GRB, typically associated with the death of massive stars ${ }^{\square}$, with an isotropic energy release of $E^{\text {iso }}=(6.0 \pm 0.1) \times 10^{53} \mathrm{erg}(50$ $300 \mathrm{keV}$ ). Observations of this GRB took place using the Swift X-ray Telescope (XRT), identifying a bright afterglow which remained detectable until almost 30 days after $T_{0}{ }^{[10] 11}$ (Fig. 1). In terms of energy flux of the X-ray afterglow $\left(0.3-10 \mathrm{keV}\right.$, at $\left.T_{0}+11 \mathrm{~h}\right)$, this GRB ranks second after the exceptional GRB 130427A ${ }^{3}$.

Observations with the H.E.S.S. array began at $T_{0}+10.1 \mathrm{~h}$ and lasted for two hours. The data were analysed using methods optimised for the detection of the lowest energy events revealing a new gamma-ray source (Fig. 2.a) with an excess of 119 gamma-ray events and a statistical significance of $5.3 \sigma$ (5.0 $\sigma$ post-trials) (see Methods). The gamma-ray excess is well fit by a point-like source model centred at right ascension $00^{\mathrm{h}} 02^{\mathrm{min}} 07.6^{\mathrm{s}}$ and declination $-02^{\circ} 56^{\prime} 06^{\prime \prime}(\mathrm{J} 2000)$ with a statistical uncertainty of $1.31^{\prime}$, consistent with the measurements at other wavelengths ${ }^{61812}$. In order to rule out the association of this source with an unknown steady gamma-ray emitter, such as an active galactic nucleus, or persistent systematic effects, the GRB region was re-observed under similar conditions 18 days after these observations. In total $6.75 \mathrm{~h}$ of data were analysed, resulting in a sky-map consistent with background events (Fig. 2.b).

The flux spectrum detected by H.E.S.S. $(100-440 \mathrm{GeV})$ was fit with a function of the form $F_{\text {obs }}(E)=F_{\text {int }}(E) \times e^{-\tau(E, z)}$, with the exponential term accounting for the absorption of photons by the extragalactic background light ${ }^{[13}$ and $F_{\text {int }}(E)=F_{0 \text {,int }}\left(E / E_{0, \text { int }}\right)^{-\gamma_{\text {int }}}$ a power-law describing the intrinsic source emission. The analysis resulted in a photon index $\gamma_{\text {int }}=1.6 \pm 1.2$ (stat.) \pm 0.4 (syst.) and a flux normalisation, evaluated at an energy $E_{0 \text {,int }}=0.154 \mathrm{TeV}$, of $F_{0, \text { int }}=(7.52 \pm$ 2.03 (stat.) ${ }_{-3.84}^{+4.53}$ (syst.) $) \times 10^{-10} \mathrm{TeV}^{-1} \mathrm{~cm}^{-2} \mathrm{~s}^{-1}$ (see Methods).

The very-high-energy (VHE) flux together with measurements at other wavelengths is shown in Fig.1. Apart from the exceptional flux level, the light curves show a typical power-law behaviour in the X-ray and optical afterglow with a temporal flux decay of the form $F(t) \propto t^{-\alpha}$ with $\alpha_{\mathrm{XRT}}=$ $1.29 \pm 0.01$ and $\alpha_{\text {optical }}=1.24 \pm 0.02$. The spectrum measured by the Fermi-LAT (100 MeV$10 \mathrm{GeV}$ ) from $T_{0}+55 \mathrm{~s}$ to $T_{0}+700 \mathrm{~s}$ is well fit by a power-law model with photon index $\gamma_{\mathrm{LAT}}=$ $2.10 \pm 0.10$. The light curve in the same time window is fit by a power-law with a temporal decay index $\alpha_{\mathrm{LAT}}=1.83 \pm 0.25$. It is worth noting that $\alpha_{\mathrm{LAT}}$ is at $\sim 1 \sigma$ from the mean value of the distribution of the decay indices of long GRBs detected by the Fermi-LAT ${ }^{[14}\left(\bar{\alpha}_{\mathrm{LAT}}=0.99 \pm 0.04\right.$, $\left.\sigma_{\bar{\alpha}}=0.80 \pm 0.07\right)$ and such deviation could largely depend on the time range where $\alpha_{\mathrm{LAT}}$ is fitted, potentially in agreement with $\alpha_{\mathrm{XRT}}$ and $\alpha_{\text {optical }}$.

The detection of VHE gamma-ray emission indicates the presence of very energetic particles 
in the GRB afterglow. This discovery is consistent with efficient gamma-ray emission seen in other astrophysical sources with relativistic plasma outflow, e.g. pulsar wind nebulae or jets emerging from the nuclei of active galaxies. In the case of a GRB afterglow, the particle acceleration likely occurs at the forward shock ${ }^{15}$ (the compression shock wave propagating through the circumburst material) which should be capable of efficient electron and proton acceleration. As proton radiation processes are characterised by long energy-loss timescales relative to the dynamical timescale, the detected gamma-ray emission is more likely produced by accelerated electrons (see Methods). Therefore, two radiation processes are the most plausible dominant contributions to the VHE spectrum: synchrotron emission of an electron population in the local magnetic field ${ }^{16}$ and synchrotron self-Compton (SSC) scattering ${ }^{17 / 18}$. In the latter case, the synchrotron photons, thought to dominate the target radiation ${ }^{19}$, are inverse-Compton scattered to higher energies by the same electron population.

The SSC and synchrotron emission-origin scenarios ${ }^{[2}$ place distinctly different demands on the source acceleration efficiency. While an SSC origin requires only multi GeV energy electrons, a synchrotron origin requires an extreme accelerator potentially accelerating beyond $\mathrm{PeV}$ energies ${ }^{20}$ (see Methods). Furthermore, for GRBs to operate as $10^{20} \mathrm{eV}$ cosmic-rays sources, they must achieve extreme acceleration ${ }^{21}$. One key distinguishing characteristic between these two emission origins is that SSC predicts the presence of two bumps in the spectral-energy distribution, whose ratio depends on the energy densities in both the electrons and magnetic field, while a synchrotron model implies only a broad single component. A second difference between these processes is the maximum photon energy achievable.

Considering a synchrotron origin of the broadband afterglow energy spectrum, the highest energy for synchrotron emission from electrons in a maximally efficient accelerator is ${ }^{22} E_{\text {sync }}^{\max }=$ $9 \Gamma m c^{2} / 4 \alpha_{\mathrm{F}} \approx 100 \Gamma \mathrm{MeV}$ (with $\alpha_{\mathrm{F}}$ the fine structure constant and $\Gamma$ the bulk Lorentz factor of the forward shock). Thus, for electron-synchrotron emission to reach energies beyond $100 \mathrm{GeV}$, $10 \mathrm{hrs}$ after the prompt emission, a late-time $\Gamma$ in excess of 1000 appears required. Such a scenario is difficult to realise, with robust expectations suggesting a value $\Gamma \sim 20$ at $10 \mathrm{~h}$ (see Methods). Alternatively, circumvention of this synchrotron maximum energy limit is possible for scenarios in which either the coherence length of the magnetic turbulence is very small, or different magnetic field strengths are present in the acceleration and emission zones, or some non-ideal process is responsible for the particle acceleration (see Methods). Regardless of this challenge, this could explain the similarity in the photon index and level of energy flux of the emission seen both at early times by Fermi-LAT and Swift-XRT and at late times by H.E.S.S. and Swift-XRT (Fig. 1). However, the strong requirements for synchrotron emission to extend up to the VHE regime disfavours such origin and the potential onset of a new SSC component should be considered.

The SSC scenario has the advantage that the emission up to VHE at late times is energetically much more easily achievable ${ }^{23}$, leading to the expectation of a new spectral component at VHE. The H.E.S.S. spectral fit constraints (Extended Data Fig. 1) are consistent with such a possibility within present uncertainties. Despite this advantage, the potential onset of inverse Compton emission within the Klein-Nishina $(\mathrm{KN})$ regime faces challenges (see Methods). Specifically, be- 
yond the gamma-ray energy where this sets in, a softer spectral slope and a different brightness evolution of this component ${ }^{24}$ are expected. However, interestingly, the presence of synchrotron emission with a hard photon index extending below keV energies can delay the onset of the full $\mathrm{KN}$ transition sufficiently to higher energies (see Methods), beyond that of the very-high energy detection. The detection of this hard extended synchrotron emission component thus delivers additional supporting evidence for an SSC origin.

This VHE discovery undoubtedly opens a key channel to the understanding of the GRBs afterglow phenomena. This measurement proves complementary to the VHE-afterglow emission detected in GRB 190829A ${ }^{25}$ and the prompt-to-early afterglow emission measured in GRB 190114C by the MAGIC telescopes ${ }^{26}$, providing insight into the nature of GRBs and their VHE detectability. We estimate that future instruments, such as the Cherenkov Telescope Array ${ }^{27}$, will allow up to 3 more GRB afterglow detections per year in the VHE domain than previously anticipated (see Methods), significantly improving our understanding of GRBs over a diverse range of timescales. 


\section{References}

1. Mészáros, P. Gamma-ray bursts. Reports on Progress in Physics 69, 2259-2321 (2006).

2. Zhang, B. \& Mészáros, P. Gamma-Ray Bursts: progress, problems \& prospects. International Journal of Modern Physics A 19, 2385-2472 (2004).

3. Ackermann, M. et al. Fermi-LAT Observations of the Gamma-Ray Burst GRB 130427A. Science 343, 42-47 (2014).

4. Piron, F. Gamma-ray bursts at high and very high energies. Comptes Rendus Physique 17, 617-631 (2016).

5. Roberts, O. J. et al. GCN22981 - GRB 180720B: Fermi-GBM observation. GCN Circulars (2018).

6. Siegel, M. H. et al. GCN22973 - GRB 180720B: Swift detection of a burst. GCN Circulars (2018).

7. Malesani, D. et al. GCN22996 - VLT/X-shooter redshift. GCN Circulars (2018).

8. Bissaldi, E. et al. GCN22980 - GRB 180720B: Fermi-LAT detection. GCN Circulars (2018).

9. Levan, A. et al. Gamma-Ray Burst Progenitors. Space Sci. Rev. 202, 33-78 (2016).

10. Evans, P. A. et al. Methods and results of an automatic analysis of a complete sample of Swift-XRT observations of GRBs. Mon. Not. R. Astron. Soc. 397, 1177-1201 (2009).

11. Evans, P. A. et al. An online repository of Swift/XRT light curves of $\gamma$-ray bursts. Astron. Astrophys. 469, 379-385 (2007).

12. Schmalz, S. et al. GCN23020 - ISON-Castelgrande observation of GRB 180720B. GCN Circulars (2018).

13. Franceschinin, A., Rodighiero, G. \& Vaccari, M. Extragalactic optical-infrared background radiation, its time evolution and the cosmic photon-photon opacity. Astron. Astrophys. 487, $837-852$ (2008).

14. Ajello, M. et al. A Decade of Gamma-Ray Bursts Observed by Fermi-LAT: The Second GRB Catalog. Astrophys. J. 878, 52 (2019).

15. Chevalier, R. A. \& Li, Z. Y. Wind Interaction Models for Gamma-Ray Burst Afterglows: The Case for Two Types of Progenitors. Astrophys. J. 536, 195-212 (2000).

16. Kumar, P. \& Barnoil Duran, R. On the generation of high-energy photons detected by the Fermi Satellite from gamma-ray bursts. Mon. Not. Roy. Astron. Soc. 400, L75-L79 (2009).

17. Sari, R. \& Esin, A. A. On the Synchrotron Self-Compton Emission from Relativistic Shocks and Its Implications for Gamma-Ray Burst Afterglows. Astrophys. J. 548, 787-799 (2001). 
18. Zhang, B. \& Mészáros, P. High-Energy Spectral Components in Gamma-Ray Burst Afterglows. Astrophys. J. 559, 110-122 (2001).

19. Warren, D. C et al. Nonlinear particle acceleration and thermal particles in GRB afterglows. Astrophys. J. 835, 248 (2017).

20. Guilbert, P. W., Fabian, A. C. \& Rees, M. J. Spectral and variability constraints on compact sources. Mon. Not. R. Astron. Soc. 205, 593-603 (1983).

21. Aharonian, F. A., Belyanin, A. A., Derishev, E. V., Kocharovsky, V. V. \& Kocharovsky, VI. V. Constraints on the extremely high-energy cosmic ray accelerators from classical electrodynamics. Phys. Rev. D 66, 023005 (2002).

22. Aharonian, F. A. TeV gamma rays from BL Lac objects due to synchrotron radiation of extremely high energy protons. New Astronomy 5, 377-395 (2000).

23. Warren, D. C., Barkov, M. V., Hirotaka, I., Nagataki, S. \& Laskar, T. Synchrotron selfabsorption in GRB afterglows: the effects of a thermal electron population. Mon. Not. Roy. Astron. Soc. 480, 4060 (2018).

24. Nakar, E., Ando, S. \& Sari, R. Klein-Nishina Effects on Optically Thin Synchrotron and Synchrotron Self-Compton Spectrum. Astrophys. J. 703, 675-691 (2009).

25. de Naurois, M. et al. GRB190829A: Detection of VHE gamma-ray emission with H.E.S.S.. The Astronomer's Telegram 13052, (2019).

26. Mirzoyan, R. First time detection of a GRB at sub-TeV energies; MAGIC detects the GRB 190114C. The Astronomer's Telegram 12390, (2019).

27. CTA Consortium Science with the Cherenkov Telescope Array. World Scientific Publishing Co (2019). 


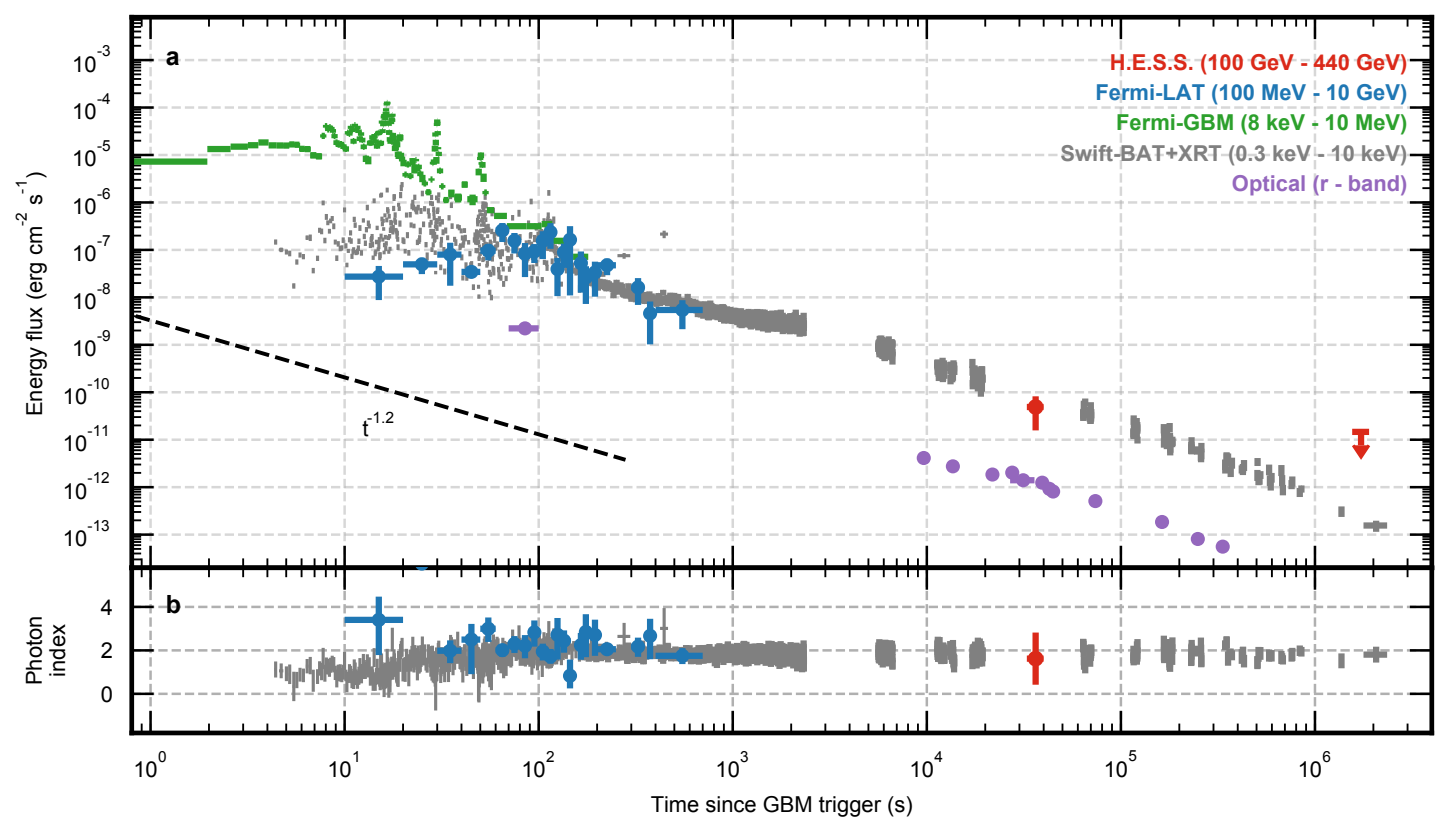

Figure 1: Multi-wavelength light curve of GRB 180720B. Panel a shows the energy flux light curve detected by the Fermi-GBM (Band fit, green), Fermi-LAT (power-law, blue), H.E.S.S energy flux and 95\% Cl L.(power-law intrinsic, red), optical r-band (purple). The Swift-BAT spectra $(15 \mathrm{keV}-150 \mathrm{keV})$ extrapolated to the XRT band (0.3-10 keV) for a combined light curve (grey). The black dashed line indicates a temporal decay with $\alpha=-1.2$. Panel $\mathbf{b}$ shows the photon index of the Fermi-LAT, Swift and H.E.S.S. spectra. Error bars correspond to $1 \sigma$ standard deviation. 

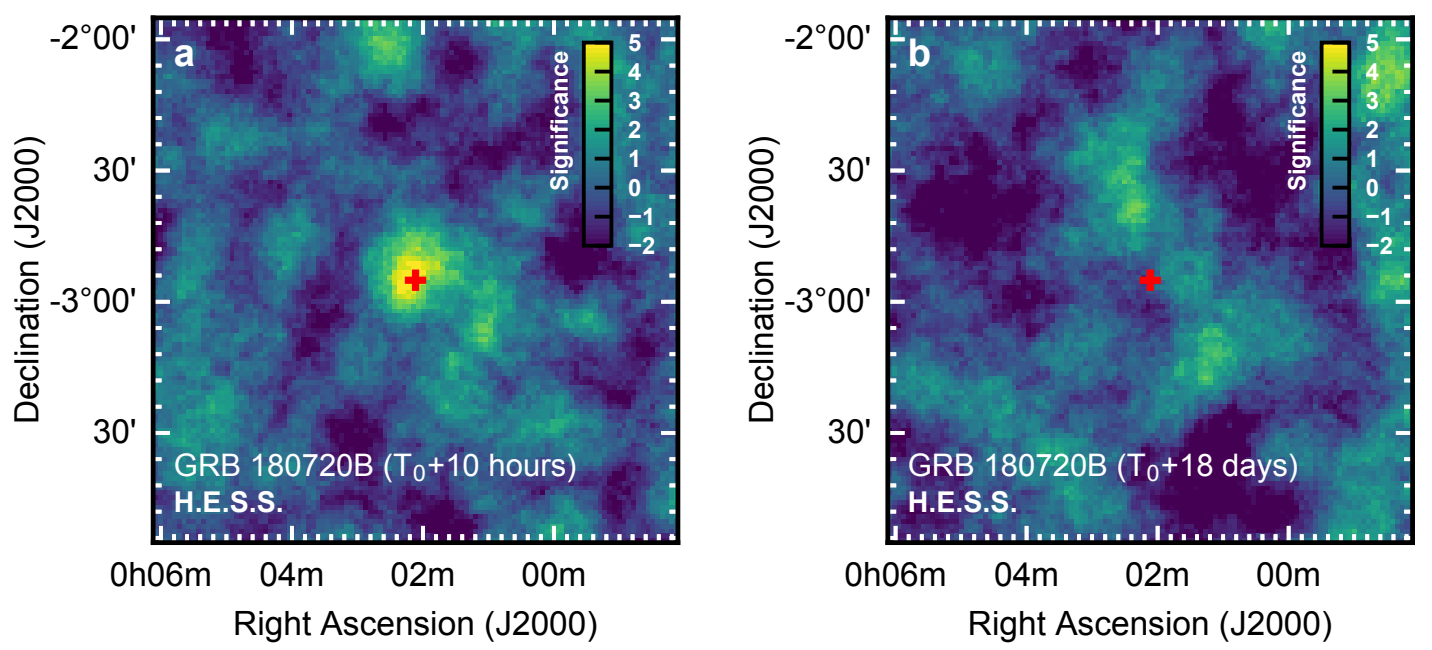

Figure 2: Very-high-energy gamma-ray image of GRB 180720B. Significance map of the GRB 180720B field as observed by H.E.S.S. Panel a corresponds to the observation made at $T_{0}+10.1 \mathrm{~h}$ for a total of $2 \mathrm{~h}$. Panel $\mathbf{b}$ shows the same region of the sky as observed during consecutive nights between $T_{0}+18.4$ days and $T_{0}+24.4$ days. The red cross indicates the position reported by the optical telescope ISON-Castelgrande ${ }^{12}$.

\section{Methods}

H.E.S.S. and the GRB follow-up programme The observations presented in this paper were performed using the H.E.S.S. array of imaging atmospheric Cherenkov telescopes situated at an altitude of $1800 \mathrm{~m}$ in the Khomas highlands of Namibia. H.E.S.S. is sensitive to gamma-rays in the energy range from tens of $\mathrm{GeV}$ to tens of $\mathrm{TeV}$. It consists of five Cherenkov telescopes, four of $108 \mathrm{~m}^{2}$ in mirror area placed on a square configuration of side length $120 \mathrm{~m}$ (CTs 1-4) and a single telescope at the centre (CT5) with a mirror area of $614 \mathrm{~m}^{2}$. Thanks to its low energy threshold and fast slewing (200 degrees per minute ${ }^{28}$ ), CT5 is well suited for the observation of soft-spectrum transient sources.

H.E.S.S. maintains an active transient source observation programme, of which gamma-ray bursts (GRB) are an important component. To ensure a fast reaction to GRB alerts, H.E.S.S. is connected to the gamma-ray coordinates network $(\mathrm{GCN}) \stackrel{29}{2}$, which rapidly distributes alerts and observational information from space and ground-based facilities. The target of opportunity observation system in H.E.S.S. performs the selection, filtering and processing of these alerts based on source observability and significance, aiming to trigger on bright, precisely located, and nearby bursts. Alerts are followed-up in two different observation modes. Observations are triggered in prompt mode when the GRB position is observable from the H.E.S.S. site at the time that the alert is received. In this case, the current observation schedule is interrupted and the array is automatically re-pointed to the GRB location. On the other hand, afterglow observations take place for GRBs 
which only become observable at a later time and are scheduled manually and triggered by a burst advocate. This was the case for GRB 180720B, observed from $T_{0}+10.1 \mathrm{~h}$ when the burst position rose above $45^{\circ}$ in elevation (below this elevation GRBs are typically not observed due to the rapid increase in energy threshold). Re-observations were carried out at $T_{0}+18$ days, after the end of an intervening moonlight period.

H.E.S.S. data analysis To reach the lowest possible energies in the analysis presented here, we use only data from the single large telescope (CT5). However, this energy threshold reduction comes at the cost of some angular resolution and sensitivity loss 30 . In total we present here two hours of observations taken in wobble mode ${ }^{31}$ with the telescope pointing direction at an offset of $0.5^{\circ}$ from the position provided by Swift-BAT ${ }^{6}$. This observation was made at a mean zenith angle of $31.5^{\circ}$ for a total live-time of $1.8 \mathrm{~h}$.

In order to ensure that a potential GRB signal is not diminished by an excessive number of statistical trials, the data analysis is subject to a strict unblinding procedure. The first step in this unblinding is an inspection of the low-level data, as some calibration artefacts can directly lead to the creation of spurious sources in the field of view. Checks are made on the fractional event participation of each camera pixel (to ensure single faulty pixels do not dominate the events), the pixel pedestal values, and the distribution of events within the field of view. Once these checks are completed, with no artefacts found, the event properties are reconstructed using the ImPACT $32[33$ maximum likelihood-based fitting technique. Background cosmic-ray events are rejected using a neural network based scheme ${ }^{34}$. The residual background contamination level of the source region ( $O N$ and $O F F$ events) and the ratio of the on-source time to the off-source time $\left(\alpha_{\operatorname{Exp} .}\right)$ is then estimated using the ring method for the production of maps and reflected-region method when performing the spectral extraction ${ }^{31}$. Full analysis and checks are performed using an additional independent calibration and data analysis chain ${ }^{35}$, serving as a cross-check of all the results presented.

The source significance is computed by using a maximum-likelihood ratio test based on the number of events coming from the source $(\mathrm{ON})$ and the background $(\mathrm{OFF})$ for a given on-source to off-source time $(\alpha)^{36}$. For the ring method, the number of $\mathrm{ON}$ and OFF events is 544 and 4740 , and $\alpha_{\text {Exp. }}=0.09$ resulting in a significance of detection of $5.3 \sigma$. Similarly the reflected-region measured 544 and 3998 events, and $\alpha_{\text {Exp. }}=0.11$ for a significance of $4.6 \sigma$ which is verified by the cross-check analysis providing 651 and 5200 of ON and OFF events and $\alpha_{\text {Exp. }}=0.10$ with a significance of $4.5 \sigma$.

The source morphology is fit with a 2D-likelihood procedure by assuming point-like and Gaussian source models convolved with the expected energy-dependent point spread function (obtained from simulations) and the measured source spectrum. Both source models proved to be compatible with the morphology of the discovered source, with no significant preference for source extension shown.

Spectral analysis is performed using the forward-folding method ${ }^{37}$, which corrects for the limited energy resolution of the single-telescope event reconstruction. The measured source spec- 


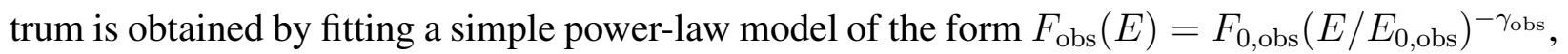
where $F_{0, o b s}$ is the flux normalisation, $\gamma_{\text {obs }}$ the photon index, and $E_{0, \text { obs }}$ the reference energy. However, due to the absorption of the most energetic photons by the extragalactic background light (EBL), the apparent photon index of this source will be somewhat steeper than the intrinsic behaviour. The intrinsic spectrum $F_{\text {int }}(E)$ is therefore obtained by fitting the measured spectrum to an attenuated power-law model $F_{\text {obs }}(E)=F_{\text {int }}(E) \times e^{-\tau(E, z)}=F_{0 \text {,int }}\left(E / E_{0, \text { Int }}\right)^{-\gamma_{\text {int }}} \times e^{-\tau(E, z)}$, where the last term in the equation corresponds to the EBL absorption coeficient predicted ${ }^{[13}$ for a redshift of 0.653. The best-fit spectra together with the spectral points are shown in Extended Data Fig. 1, and the spectral parameters are summarised in Extended Data Table 1.

Systematic uncertainties in the fitted spectra are determined accounting for a $15 \%$ uncertainty in the reconstructed energy due to possible variations in the measured Cherenkov light yield ${ }^{38}$. The measured energy is systematically shifted by $\pm 15 \%$ and the whole spectral fitting procedure is redone. In addition to this, short dips in the trigger rate, at the level of $30 \%$, were identified in the data, most likely attributed to the presence of high altitude clouds. In order to assess the effect of these, the time windows containing such trigger rate features (21.7 min total) were removed from the data and the standard analysis described above was performed on the reduced data set. From this, we conclude an additional systematic underestimation of $32 \%$ and $4.8 \%$ in the measured normalisation and photon index respectively. These two sources of systematic uncertainty are considered to be independent and therefore added in quadrature for a total systematic uncertainty estimation.

The intrinsic spectrum was obtained with a chosen EBL model ${ }^{13}$. To determine the level by which this choice influences the results presented in this work, the data were re-analysed using three additional EBL models ${ }^{[3941}$, each one employing a different approach to predict the overall EBL level ${ }^{[39}$. The absorption coefficient for a redshift of 0.653 within the energy range of the detected emission does not present significant deviations between these models considered(Extended Data Fig. 2 extended data). When employing these EBL models for the spectral fit, a change in the reported normalisation and index of maximum $55.3 \%$ and $27 \%$ respectively was found. The statistical uncertainty on the fitted spectra remains as the biggest source of uncertainty in the results.

Trial correction Since 2012, H.E.S.S. has performed five additional follow-up observations of well localised GRBs (Swift and Fermi-LAT alerts) using only CT5 (similar to the observations presented here). The significance distribution of this sample (excluding GRB 180720B) is consistent with pure statistical fluctuations. Therefore, a post-trial significance for GRB 180720B can be assessed by accounting for these GRBs previously observed. This results in a post-trial significance of $4.3 \sigma$ (reflected-region method) and $5.0 \sigma$ (ring method). As the analysis of GRB 180720B was performed once under the aforementioned unblinding procedure, no additional trials have been added to the results presented here.

Background systematic effects Systematic effects on the sky map background (Fig. 2) were determined by measuring the significance distribution when excluding the source region. While a normal distribution is expected, a width of 1.09 was measured in this significance distribution, therefore adding a slight shift to the reported significance of the ring method (used in the produc- 
tion of sky maps). The corrected significance when accounting for such effects is $4.9 \sigma$ (4.7 $\sigma$ post-trial). Nonetheless, this measured distribution highly depends on the parameters of the ring method and should be subject to statistical uncertainties.

Fermi data analysis The Fermi-GBM data for GRBs are publicly available through the GBM Burst Catalog at HEASARC ${ }^{42}$. For GRB 180720B the available time-tagged events (TTE) of those detectors having the best viewing angle to the Swift-XRT position, namely n6, n7, nb, and b1, were analysed. Temporally-resolved energy flux data points (Fig. 1) were obtained with the RMfit analysis software ${ }^{433}$ by combining TTE data from all four detectors into $256 \mathrm{~ms}$ bins in the energy range from $8 \mathrm{keV}$ to $10 \mathrm{MeV}$.

The Fermi-LAT data analysis was performed using the "Pass 8 " ${ }^{47}$ processed events. We used the P8R3_TRANSIENT010E event class that is suitable for transient source analysis, and the corresponding instrument response functions ${ }^{44}$. Events were selected from $T_{0}$ to $T_{0}+700 \mathrm{~s}$ in the standard GRB-analysis energy range from $100 \mathrm{MeV}$ to $100 \mathrm{GeV}$ over a region of $10^{\circ}$ around the Swift-XRT localisation. Event selection, quality cuts and data analysis were performed with the standard FermiTools ${ }^{45}$ software. The source detection over the full duration was determined by a likelihood analysis providing a test-statistic value of TS $\sim 600$, which corresponds to a significance $\sigma \sim 25(\sigma \sim \sqrt{T S})$.

Since the highest energy photon detected has an energy of $5 \mathrm{GeV}$ (at $T_{0}+142.4 \mathrm{~s}$ ) the temporally-resolved energy flux data points (Fig. 1) were computed in the energy range from 100 $\mathrm{MeV}$ to $10 \mathrm{GeV}$. The analysis model included the Galactic interstellar emission model (gll_iem_v06.fits) and the relative isotropic diffuse emission templates provided by the Fermi-LAT collaboration ${ }^{48}$, where the normalisation of the latter was left free to vary. The spectrum for each bin was fit to a single power-law model $F(E)=F_{0} \times\left(\frac{\mathrm{E}}{\mathrm{E}_{0}}\right)^{-\gamma}$ having the flux normalisation $F_{0}$ and the photon index $\gamma$ as free parameters. As no emission with energies $>10 \mathrm{GeV}$ is detected, no additional term in the spectra accounting for EBL absorption ${ }^{13}$ was required. The temporal decay $\alpha_{\mathrm{LAT}}$ was fit to a power-law model using a least-squares technique applied from $T_{0}+55 \mathrm{~s}$ to $T_{0}+700 \mathrm{~s}$, in order to assure no contamination of the prompt emission as observed by the Fermi-GBM and Swift-BAT, obtaining a reduced $\chi^{2}=0.63($ d.o.f. $=14)$.

Optical data The optical data shown in Fig. 1 were compiled from the GCN circulars of observations performed in the r-band by the following instruments: Kanata ${ }^{49}$, MITSuME ${ }^{50}$, TSHAO ${ }^{51}$, MASTER-K ${ }^{52}$, MASTER-I, ISON-Castelgrande ${ }^{[12}$, OSN ${ }^{53}$, LCO $^{54}$, and KAIT ${ }^{55}$. The reported temporal decay index $\alpha_{\text {optical }}$ is measured from $T_{0}+9642 \mathrm{~s}$ to $T_{0}+3.35 \times 10^{5} \mathrm{~s}$ by performing a power-law fit with a $\chi^{2}$ fitting procedure.

Swift data The Swift data are publicly available through the Swift online repository ${ }^{46}$. The temporally resolved energy-flux data shown in Fig. 1 were obtained using the Burst Analyser tool ${ }^{10,11}$. Data are rebinned for a signal-to-noise ratio (SNR) of 7 and systematic uncertainties are included. The temporal decay reported here $\left(\alpha_{\mathrm{XRT}}\right)$ is obtained from $T_{0}+2200 \mathrm{~s}$ to $T_{0}+3.05 \times 10^{5} \mathrm{~s}$ and corresponds to the fourth break in the light curve as identified from the fitting procedures of the Swift-XRT tools. 
CTA detectability prospects Considering CTA to be an order of magnitude more sensitive than the H.E.S.S. array implies the ability to detect energy fluxes $\sim 10$ times fainter than that of GRB 180720B at VHE. If the VHE energy flux equals that detected by Swift-XRT, as suggested by our measurements (Fig. 1), we estimate an occurrence of 3 GRBs per year above such assumed flux and therefore detectable by CTA (Extended Data Fig. 3). Such number could be increased for follow-up observations at earlier times. By assuming a temporal decay value $\alpha=1.2\left(F(t) \propto t^{-\alpha}\right)$ for all the GRB afterglows detected by Swift-XRT ${ }^{56}$ an extrapolation of the 11 hours energy flux to the one expected at 5 hours provides a detectability prospect of $\sim 10$ GRBs per year at such follow-up delay times. It should be noted however, that the presence of VHE emission could also be dependent on the GRB environment ${ }^{[57}$ and such influence is not considered in this estimation.

The Bulk Lorentz Factor depends on two factors: the released energy and the density of the circumburst medium ${ }^{58}, \Gamma=\sqrt{E^{\text {iso }} / M c^{2}}$, where $E^{\text {iso }}$ is the equivalent isotropic energy and $M$ is the total mass swept-up by the shock. The latter depends on the nature of the circumburst environment: $M=(4 \pi / 3) R^{3} n m_{p}$ for a homogeneous medium (here $n$ is the medium number density and $R$ is the shock radius) or $M=\dot{M}_{*} R / v_{*}$ for a shock propagating in a constant velocity wind (here $\dot{M}_{*}$ and $v_{*}$ are the wind mass-loss rate and velocity, respectively). The shock radius depends on the detection time as $R \simeq A_{\mathrm{R}} \Gamma^{2} t c /(1+z)$, where $A_{\mathrm{R}}=8$ and 4 for homogeneous medium and wind environments, respectively. Thus, for GRB 180720B ( $t=10 \mathrm{~h}, z=0.653$, and $E^{\text {iso }} \simeq 10^{54} \mathrm{erg}$ ) one obtains $\Gamma=15 n_{0}^{-1 / 8}$ (here $n_{0}=n / \mathrm{cm}^{-3}$ ) for a homogeneous medium or $\Gamma=20 \dot{M}_{*,-5}^{-1 / 4} v_{*, 3.3}^{1 / 4}\left(\right.$ here $\dot{M}_{*,-5}=\dot{M}_{*} /\left(10^{-5} M_{\text {sun }} / \mathrm{yr}\right)$ and $\left.v_{*, 3.3}=v_{*} /(2000 \mathrm{~km} / \mathrm{s})\right)$ for a wind environment.

Non-thermal process efficiency,$\kappa=t_{\mathrm{dyn}} / t_{\mathrm{cool}}$, depends on the ratio of the shock dynamic time-scale, $t_{\mathrm{dyn}}=R /(c \Gamma)$, to the cooling time, $t_{\mathrm{cool}}$, which depends on radiation mechanism, density of the target, and the energy of the parent particles. For hadronic processes $\underset{59}{ }$, which include proton-proton $(p p)$ and photon-meson $(p \gamma)$ channels, the radiation efficiency is $\kappa_{p p} \sim$ $10^{-7}\left(R / 10^{18} \mathrm{~cm}\right) n_{0}$ and $\kappa_{p \gamma} \sim 3 \times 10^{-4}(\Gamma / 20)^{2}\left(R / 10^{18} \mathrm{~cm}\right)\left(\kappa / 10^{-2}\right)\left(E_{\mathrm{t}} / 1 \mathrm{keV}\right)^{-1} n_{0}$ (here $\kappa$ is the total radiative efficiency, and $E_{\mathrm{t}}$ is the peak frequency of the soft emission component). These low efficiencies favor the electromagnetic processes 60 . The efficiency of the synchrotron channel for the emission detected in the VHE band, $E \sim 100 \mathrm{GeV}$, is $\kappa_{\text {sync }}=5 \times$ $10^{7}\left(m_{e} / m\right)^{5 / 2}\left(R / 10^{18} \mathrm{~cm}\right)\left(\eta_{\mathrm{B}} / 0.1\right)^{3 / 4} \eta_{\text {turb }}^{-1 / 2} n_{0}^{3 / 4}$, where $m_{e}$ and $m$ are electron and emitting particle masses; $\eta_{\mathrm{B}}$ is a fraction of the internal energy contained in the magnetic field; and $\eta_{\text {turb }}=$ $\max \left(1, R_{\mathrm{Lar}}^{\mathrm{nr}} / \lambda_{\text {cor }}\right)$ defines the shift of the peak energy if a charged particle interacts with turbulent magnetic field ${ }^{61}$ (here $R_{\mathrm{Lar}}^{\mathrm{nr}}$ is the non-relativistic Larmor radius and $\lambda_{\text {cor }}$ is the magnetic field correlation length). If the inverse Compton scattering proceeds in the Thomson regime, then $\kappa_{\mathrm{IC}}=3(\Gamma / 20)\left(\kappa / 10^{-2}\right)\left(R / 10^{18} \mathrm{~cm}\right)\left(E_{\mathrm{t}} / 1 \mathrm{keV}\right)^{-1 / 2} n_{0}$. Efficiencies larger than 1 indicate that particle cooling occurs faster than the source dynamical timescale, and is therefore highly efficient.

Synchrotron emission is characterised by the highest radiation efficiency, but this emission component peaks below this limiting energy: $E_{\mathrm{sync}}^{\max }=100 \Gamma\left(\mathrm{m} / m_{e}\right) \eta_{\mathrm{turb}}\left(B_{\mathrm{em}} / B_{\mathrm{ac}}\right)\left(E_{\mathrm{ac}} / B_{\mathrm{ac}}\right) \mathrm{MeV}$. Here $B_{\mathrm{em}}$ and $B_{\mathrm{ac}}$ are magnetic field strengths at the emitter and accelerator sites, respectively. The accelerating electric field, $E_{\mathrm{ac}}$, is smaller than the magnetic field, $E_{\mathrm{ac}}<B_{\mathrm{ac}}$, if the particle accel- 
eration proceeds in the ideal magnetohydrodynamic (MHD) flows ${ }^{21}$. Thus, the production of VHE gamma rays via electron synchrotron emission requires a large Lorentz factor, $\Gamma>10^{3}$; a very small-scale magnetic turbulence, $\lambda_{\text {cor }}<10^{-2} R_{\mathrm{Lar}}^{\mathrm{nr}}$; a large change of the magnetic field strength, $B_{\text {em }}>10^{2} B_{\text {ac }}$; particle acceleration to operate in the non-ideal MHD regime; or a combination of these factors. Proton synchrotron alleviates these requirements in expense of a significantly lower radiation efficiency. Whilst proton synchrotron emission out-competes other hadronic radiation

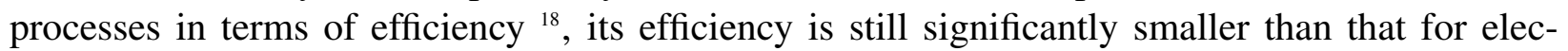
trons. Thus proton synchrotron emission is expected to only give rise to a subdominant emission component within the VHE band.

The energy of particles emitting in the VHE regime depends on the dominant radiation mechanism and ejecta properties. In the case of a synchrotron origin scenario the particle energy is determined by three important factors: the shock Lorentz factor, the strength of the magnetic field, and the turbulence scale. While the first factor, $\Gamma \simeq 20$, is relatively well defined by the epoch of the H.E.S.S. observation, the magnetic field strength and possibility of the realisation of smallscale turbulence remains highly uncertain. The internal energy density, $\sim 0.1(\Gamma / 20)^{2} n_{0} \mathrm{~J} / \mathrm{m}^{3}$, suggests that a Gauss-strength magnetic field is expected for the case of energy equipartition between the magnetic field and particles. We note, however, that significantly smaller plasma magnetisation is discussed in the literature ${ }^{62}$, corresponding to several orders of magnitude weaker magnetic field strengths. Assuming that synchrotron emission beyond the $100 \mathrm{MeV}$ energy limit in co-moving frame can be achieved, the energy of the emitting electrons can be estimated as $E_{e} \simeq 4(E / 100 \mathrm{keV})^{1 / 2}(\Gamma / 20)^{-1 / 2}(B / 0.1 \mathrm{G})^{-1 / 2} \eta_{\text {turb }}^{-1 / 2} \mathrm{TeV}$. The production of $100 \mathrm{GeV}$ gamma rays through a synchrotron scenario therefore requires electrons of ultra-high-energy, $E_{e} \sim 4 \mathrm{PeV}$, unless a configuration with a very small-scale turbulence is present. The energy of particles that provide the dominant contribution to the IC emission depends strongly on the spectrum of the target photons and bulk Lorentz factor. An electron with energy $E_{e}$ up-scatters a target photon with energy $E_{\mathrm{t}}$ to energy $\min \left(E_{\mathrm{t}}\left(E_{e} / m_{e} c^{2}\right)^{2}, \Gamma E_{e}\right)$. For target photons detected in the X-ray energy band $E_{\mathrm{t}} \sim 1 \mathrm{keV}$, electrons with energy $E_{e} \sim 10 \mathrm{GeV}$, which in the lab frame have an energy of hundred of $\mathrm{GeV}$, can produce gamma rays that are detected in the VHE energy band.

Target photons of very different energies can be up-scattered to gamma rays of the same energy. This can be of particular relevance for VHE detected from GRBs, with both target photons and nonthermal electrons likely possessing broad energy distributions. Assuming a power-law distribution for the target photon flux, $d n / d E_{t} \propto E_{t}^{-\gamma}$, and for electrons, $d n_{\mathrm{e}} / d E_{e} \propto E_{e}^{-\gamma_{\mathrm{e}}}$ one finds that the relative contribution to the gamma-ray emission depends on the electron energy as $\propto(1-$ $\left.E /\left(E_{e} \Gamma\right)\right) E_{e}^{2 \gamma-\gamma_{e}}$. For the sake of simplicity, just a single high-energy term in the cross-section was accounted for (resulting in the factor $1-E /\left(E_{e} \Gamma\right)$ ), sufficient for a qualitative study. However, the obtained dependence shows that for a reasonable range of photon and electron indices, $1.5<$ $\gamma, \gamma_{\mathrm{e}}<3$, the highest-available-energy electrons may provide an important contribution to the gamma-ray energy band by up-scattering photons with energies within the infrared (IR) to ultraviolet (UV) range. 
The Klein-Nishina cutoff is a significant reduction of the Compton cross-section that occurs when $E_{e} E_{\mathrm{t}} \gtrsim \Gamma m_{e}^{2} c^{4}$, where $E_{e}$ and $E_{\mathrm{t}}$ are the electron and target photon energies in the comoving frame and laboratory systems, respectively. This results in a softening of the gamma-ray spectrum that occurs for $E \gtrsim 50(\Gamma / 20)^{2}\left(E_{\mathrm{t}} / 1 \mathrm{keV}\right)^{-1} \mathrm{GeV}$. Since typically GRB synchrotron spectral energy distribution peaks in the $\mathrm{keV}$ band, the inverse Compton component detected at late afterglow phases may be affected by the Klein-Nishina cutoff, resulting in reduced fluxes and steeper spectra. This may appear to be in contradiction with a relatively hard intrinsic spectral index, $\gamma_{\text {int }} \sim 1.6$ inferred from the H.E.S.S. measurement. There are, however, two effects that can result in a spectral hardening at energies around the cutoff: (i) the up-scattering of low energy IR to UV photons, giving an intrinsic VHE component with the same slope as seen in the hard Xray band; (ii) and hardness of the electron spectrum at $\mathrm{GeV}$ energies, where adiabatic losses likely render the electron spectrum hard. The search for consistency within this framework of the hard VHE spectrum with the SSC scenario, however, requires detailed dedicated simulations, which are beyond the scope of this observational paper. 


\section{References}

28. Hofverberg, P., Kankanyan, R., Panter, M., Hermann, G., Hofmann, W., Deil, C. \& Benkhali, F. A. Commissioning and initial performance of the H.E.S.S. II drive system. PoS ICRC2013, 926 (2013).

29. Bathelmy, S. The gamma-ray burst coordinates network. http:/gcn.gsfc.nasa.gov (2019).

30. Holler, M. et al. Observations of the Crab Nebula with H.E.S.S. Phase II. PoS ICRC2015, 947 (2016).

31. Berge, D., Funk, S. \& Hinton, J. Background Modelling in Very-High-Energy gamma-ray Astronomy. Astron. Astrophys. 466, 1219-1229 (2007).

32. Parsons, R. D. \& Hinton, J. A. A Monte Carlo Template based analysis for Air-Cherenkov Arrays. Astroparticle Phys. 56, 26-34 (2014).

33. Parsons, R. D., Murach, T. \& Gajdus, M. HESS II Data Analysis with ImPACT. PoS ICRC2015, 826 (2015).

34. Murach, T., Gajdus, M. \& Parsons, R. D. A Neural Network-Based Monoscopic Reconstruction Algorithm for H.E.S.S. II. PoS ICRC2015, 1022 (2015).

35. de Naurois, M. \& Rolland, L. A high performance likelihood reconstruction of $\gamma$-rays for imaging atmospheric Cherenkov telescopes. Astroparticle Phys. 32, 231-252 (2009).

36. Li, T. P. \& Ma, Y. Q. Analysis methods for results in gamma-ray astronomy. Astrophys. J. 272, 317-324 (1983).

37. Piron, F. et al. Temporal and spectral gamma-ray properties of Mkn 421 above $250 \mathrm{GeV}$ from CAT observations between 1996 and 2000. Astron. Astrophys. 374, (2001).

38. Abdalla, H. et al. Gamma-ray blazar spectra with H.E.S.S. II mono analysis: The case of PKS 2155-304 and PG 1553+113. Astron. Astrophys. 600, A89 (2017).

39. Domínguez, A. et al. Extragalactic background light inferred from AEGIS galaxy-SED-type fractions. Mon. Not. Roy. Astron. Soc. 410, 2556-2578 (2011).

40. Finke, J. D., Razzaque, S. \& Dermer, C. D. MODELING THE EXTRAGALACTIC BACKGROUND LIGHT FROM STARS AND DUST. Astrophys. J. 712, 238-249 (2010).

41. Gilmore, R. C. et al. Semi-analytic modelling of the extragalactic background light and consequences for extragalactic gamma-ray spectra. Mont. Not. Roy. Astron. Soc. 422, 3189-3207 (2012).

42. FERMIGBRST - Fermi GBM Burst Catalog. https://heasarc.gsfc.nasa.gov/W3Browse/fermi/fermigbrst.html , (2019). 
43. GBM Software Tools. https://fermi.gsfc.nasa.gov/ssc/data/analysis/rmfit/, (2019).

44. Fermi LAT Performance. http://www.slac.stanford.edu/exp/glast/groups/canda/lat_Performance.htm , (2019).

45. Fermitools-conda-recipe. https://github.com/fermi-lat/Fermitools-conda/, (2019).

46. The Swift Burst Analyser GRB 180720B. https://www.swift.ac.uk/burst_analyser/00848890/ , (2018).

47. Atwood, W. et al. Pass 8: Toward the Full Realization of the Fermi-LAT Scientific Potential. 2012 Fermi Symposium proceedings - eConf C121028, (2013).

48. Acero, F. et al. Development of the Model of Galactic Interstellar Emission for Standard Point-Source Analysis of Fermi Large Area Telescope Data. Astrophys. J. Suppl. 223, 26 (2016).

49. Sasada, M. et al. GCN22977 - Kanata observation . https://gcn.gsfc.nasa.gov/gcn3/22977.gcn3, GCN Circulars (2018).

50. Itoh, R. et al. GCN22983 - MITSuME Akeno observation. https://gcn.gsfc.nasa.gov/gcn3/22983.gcn3, GCN Circulars (2018).

51. Reva, I. et al. GCN22979 - TSHAO observation. https://gcn.gsfc.nasa.gov/gcn3/22979.gcn3, GCN Circulars , (2018).

52. Lipunov, V. et al. GCN23023 - MASTER observation. https://gcn.gsfc.nasa.gov/gcn3/23023.gcn3, GCN Circulars , (2018).

53. Kann, D. et al. GCN22985 - OSN observation. https://gcn.gsfc.nasa.gov/gcn3/22985.gcn3, GCN Circulars , (2018).

54. Martone, R. et al. GCN22976 - LCO Haleaka observation of GRB $180720 B$. https://gcn.gsfc.nasa.gov/gcn3/22976.gcn3, GCN Circulars , (2018).

55. Zheng, W. et al. GCN23033 - KAIT observation of GRB 180720B. https://gcn.gsfc.nasa.gov/gcn3/23033.gcn3, GCN Circulars , (2018).

56. Nousek, J. A. et al. Evidence for a canonical GRB afterglow light curve in the Swift/XRT data. Astrophys. J. 642, 389-400 (2006).

57. Vurm, I. \& Beloborodov, A. M. On the prospects of gamma-ray burst detection in the TeV band. Astrophys. J. 846, 152 (2006).

58. Blandford, R. D. \& McKee, C. F. Fluid dynamics of relativistic blast waves. Physics of Fluids 19, 1130-1138 (1976). 
59. Aharonian, F. A. Very high energy cosmic gamma radiation : a crucial window on the extreme Universe. World Scientific Publishing Co , (2004).

60. Blumenthal, G. R. \& Gould, R. J. Bremsstrahlung, Synchrotron Radiation, and Compton Scattering of High-Energy Electrons Traversing Dilute Gases. Reviews of Modern Physics $\mathbf{4 2}$, 237-271 (1970).

61. Kelner, S. R., Aharonian, F. A. \& Khangulyan, D. On the Jitter Radiation. Astrophys. J. 774, 61 (2018).

62. Santana, R., Barniol, D. \& Kumar, P. Magnetic Fields in Relativistic Collisionless Shocks. Astrophys. J. 785, 29 (2014). 
Acknowledgements The support of the Namibian authorities and of the University of Namibia in facilitating the construction and operation of H.E.S.S. is gratefully acknowledged, as is the support by the German Ministry for Education and Research (BMBF), the Max Planck Society, the German Research Foundation (DFG), the Helmholtz Association, the Alexander von Humboldt Foundation, the French Ministry of Higher Education, Research and Innovation, the Centre National de la Recherche Scientifique (CNRS/IN2P3 and CNRS/INSU), the Commissariat a l'energie atomique et aux energies alternatives (CEA), the U.K. Science and Technology Facilities Council (STFC), the Knut and Alice Wallenberg Foundation, the National Science Centre, Poland grant no. 2016/22/M/ST9/00382, the South African Department of Science and Technology and National Research Foundation, the University of Namibia, the National Commission on Research, Science \& Technology of Namibia (NCRST), the Austrian Federal Ministry of Education, Science and Research and the Austrian Science Fund (FWF), the Australian Research Council (ARC), the Japan Society for the Promotion of Science and by the University of Amsterdam. We appreciate the excellent work of the technical support staff in Berlin, Zeuthen, Heidelberg, Palaiseau, Paris, Saclay, Tübingen and in Namibia in the construction and operation of the equipment. This work beneted from services provided by the H.E.S.S. Virtual Organisation, supported by the national resource providers of the EGI Federation.

The Fermi-LAT Collaboration acknowledges support for LAT development, operation and data analysis from NASA and DOE (United States), CEA/Irfu and IN2P3/CNRS (France), ASI and INFN (Italy), MEXT, KEK, and JAXA (Japan), and the K.A. Wallenberg Foundation, the Swedish Research Council and the National Space Board (Sweden). Science analysis support in the operations phase from INAF (Italy) and CNES (France) is also gratefully acknowledged. This work performed in part under DOE Contract DEAC02-76SF00515.

Data and Code Availability Statement The H.E.S.S. raw data and the code used in this study are not public but belong to the H.E.S.S. collaboration. All derived higher level data that are shown in plots will be made available on the H.E.S.S. collaborations web site upon publication of this study.

Data and analysis code from the Fermi-GBM and LAT instruments are publicly available. Links to the data and software are provided in the analysis section.

This work also made use of data supplied by the UK Swift Science Data Centre at the University of Leicester (http://www.swift.ac.uk/archive/).

\section{Author information}

Author contributions $\quad$ R. D. Parsons, Q. Piel, E. L. Ruiz-Velasco have analysed the H.E.S.S. data, interpreted the data and prepared the manuscript. E. Bissaldi analysed the Fermi data, interpreted the data and prepared the manuscript. C. Hoischen implemented the response system for the GRBs follow-up of H.E.S.S. We acknowledge the help of A. M. Taylor, F. Aharonian and D. Khangulyan on interpreting the results and preparing the manuscript. The whole H.E.S.S. collaboration has contributed to the publication with involvement at various stages ranging from the design, construction and operation of the instrument, to the development and maintenance of all software for data handling, data reduction and data analysis. All authors have reviewed, discussed, and commented on the present results and on the manuscript.

Competing Interests The authors declare that they have no competing financial interests.

Correspondance and request for materials should be addressed to E.R.-V. (contact.hess@ hess-experiment.eu). 

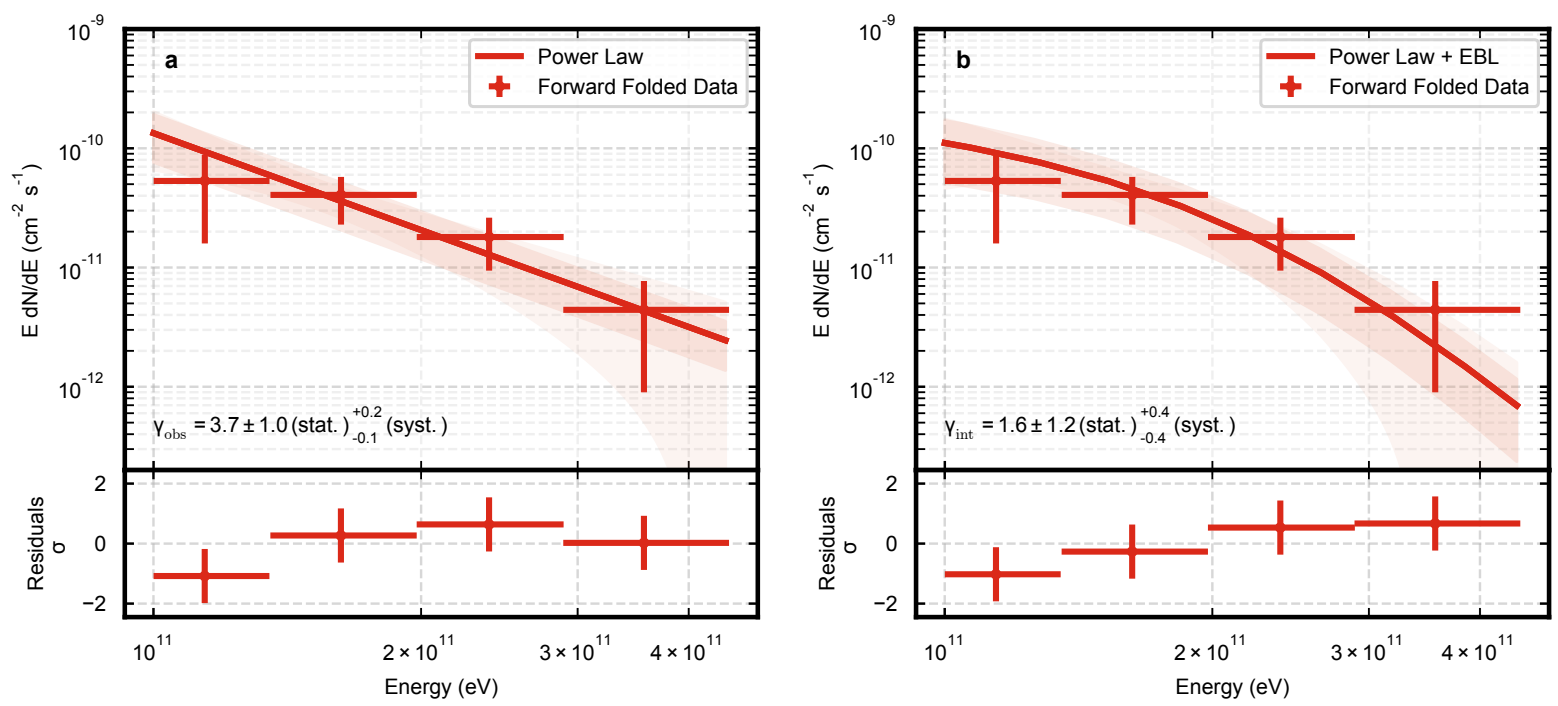

Extended Data Figure 1: VHE spectral fit of GRB 180720B. H.E.S.S. spectral fit to the measured emission in the energy range of $100-440 \mathrm{GeV}$. Panel a: Fit assuming a simple power-law model (with photon index $\gamma_{\text {obs }}$ ). Panel b: Fit assuming a power-law model (with photon index $\gamma_{\text {int }}$ ) with EBL attenuation for a source at $z=0.653$ 13. In both cases the residual data points with $1 \sigma$ uncertainties are obtained from the forward-folded method. The shaded areas show the statistical and systematic uncertainties in each fit ( $1 \sigma$ confidence level). The bottom panels show the significance of the residuals between the fitted model and the data points.

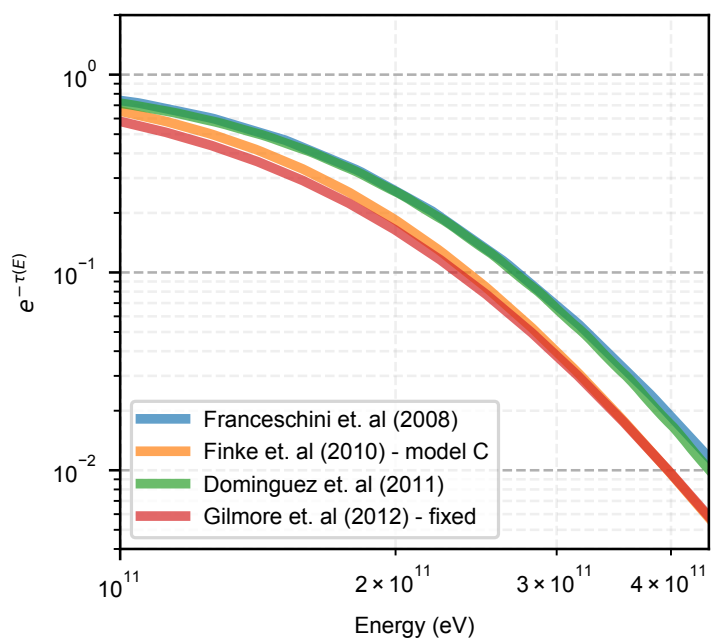

Extended Data Figure 2: EBL absorption coefficient. Absorption coefficient $e^{-\tau(E)}$ for a source emitting at a redshift 0.653. The values are shown in the energy range of the detected emission of GRB 180720B $(100-440 \mathrm{GeV})$ for the four EBL models considered $13,39,41$. 


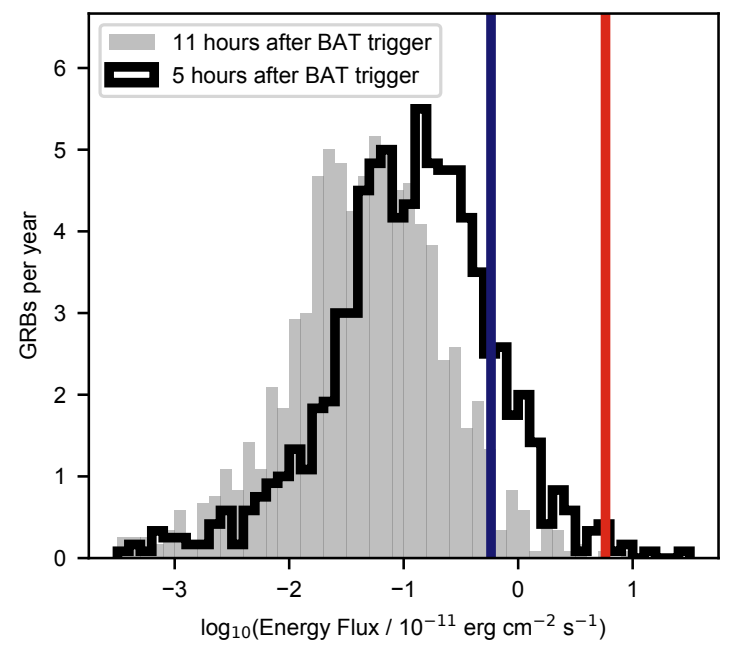

Extended Data Figure 3: CTA detectability prospects. Energy flux distribution at 11 hours and 5 hours after the SwiftBAT trigger for all the GRBs observed by the Swift-XRT per year. The blue vertical line shows the expected sensitivity of CTA, assuming the detectability of fluxes 10 times fainter than GRB 180720B. The energy flux of GRB 180720B is indicated by the red vertical line.

\begin{tabular}{lcll}
\multicolumn{5}{c}{ Extended Data Table 1 VHE Spectral information from GRB 180720B } \\
\hline Spectral model & $F_{0}\left[\times 10^{-10} \mathrm{~cm}^{-2} \mathrm{~s}^{-1} \mathrm{TeV}^{-1}\right]$ & $\gamma$ & $\mathrm{E}_{0}[\mathrm{TeV}]$ \\
\hline$F_{0} \times \frac{E}{E_{0}}-\gamma$ & $2.71 \pm 0.74_{-1.16}^{+1.43}$ & $3.7 \pm 1.0_{-0.1}^{+0.2}$ & 0.154 \\
$F_{0} \times \frac{E}{E_{0}}-\gamma \times e^{-\tau(z, E)}$ & $7.52 \pm 2.03_{-3.84}^{+4.53}$ & $1.6 \pm 1.2_{-0.4}^{+0.4}$ & 0.154 \\
$F_{0} \times \frac{E}{E_{0}}-2 \times e^{-\tau(z, E)}$ & $16.12 \pm 4.37_{-9.25}^{+10.59}$ & $2.0[$ Fixed $]$ & 0.105 \\
\hline
\end{tabular}

Spectral parameters of the fits to the H.E.S.S. observed emission in the energy range $100-440 \mathrm{GeV}$. The intrinsic spectrum with $\gamma=2.0$ (third row) is provided as a reference to the Fermi-LAT mean photon index detected in several other GRBs at HE ${ }^{14}$. All reported uncertainties are statistical and systematic in that order. 